\title{
The Imaging and Slitless Spectroscopy Instrument for Surveys (ISSIS): expected radiometric performance, operation modes and data handling
}

\author{
Ana I. Gómez de Castro • G. Belén Perea • \\ Néstor Sánchez • Javier López Santiago • \\ Jóse Chirivella $\cdot$ Juan Seijas
}

\begin{abstract}
ISSIS is the instrument for imaging and slitless spectroscopy on-board WSO-UV. In this article, a detailed comparison between ISSIS expected radiometric performance and other ultraviolet instruments is shown. In addition, we present preliminary information on the performance verification tests and on the foreseen procedures for in-flight operation and data handling.
\end{abstract}

Keywords Space vehicles: instruments - Instrumentation: spectrographs · Ultraviolet: general

\section{Introduction}

The WSO-UV (Sachkov et al. 2014) is an international project developed to guarantee access to the ultraviolet (UV) range in the post-Hubble Space Telescope era. This $170 \mathrm{~cm}$ space telescope has been conceived as a multipurpose observatory incorporating instrumentation for astronomical imaging and spectroscopy. The WSO-UV spectrographs consist of three different instruments: two highresolution ( $R \sim 55,000$ ) echelle spectrographs and a long slit spectrograph offering low resolution $(R \sim 1000)$ spectroscopy (Hermanutz et al. 2012; Sachkov 2010; Sachkov et al. 2014). The camera unit of the WSO-UV is named ISSIS: the Imaging and Slitless Spectroscopy Instrument. The baseline for ISSIS design, as approved in the PDR held in May 2012, consists of two acquisition channels, both of them provided with photon counting detectors with MicroChannel Plates (MCP). These two channels are named: the Far Ultraviolet (FUV) Channel covering the 1150-1750 A wavelength range and the Near Ultraviolet (NUV) Channel in the $1850-3200 \AA$ range.

\section{Scientific requirements}

ISSIS is aimed at providing high-spatial resolution and high UV sensitivity while trying to maximize the wavelength coverage and the size of the field of view. Slitless spectroscopy is required as an efficient tool for classification and analysis of astronomical objects. With these conditions, a list of well-defined key programs to be carried out with ISSIS was elaborated (Gómez de Castro et al. 2012a), requiring:

- High-resolution mapping of weak and nebulous sources such as micro-jets or gravitational lenses.

- The mapping of UV emission lines in extended emission nebulae (H II regions, supernovae remnants, planetary nebulae) and jets (from protostars or from compact objects).

- Efficient spectroscopy of weak sources: from transiting planets to Active Galactic Nuclei and star-forming galaxies at moderate redshifts $(0.5<z<1.5)$.

- Resolution of $R \sim 500$ to study the absorption of the stellar radiation by transiting planets or to determine the terminal velocity of radiatively driven winds of $\mathrm{O}$ stars in Local Group galaxies.

- Enhancement of the dynamic range with coronographs or masks to map faint emission close to bright sources on sub-arcsec scales: from discs to jets or binary components. 
Table 1 Point source fluxes to reach $\mathrm{S} / \mathrm{N}>10$ in $3600 \mathrm{sec}$ (imaging)

\begin{tabular}{ll}
\hline Wavelength $(\AA)$ & Flux $\left(\mathrm{erg} \mathrm{s}^{-1} \mathrm{~cm}^{-2} \AA^{-1}\right)$ \\
\hline 1500 & $9.0 \times 10^{-17}$ \\
2300 & $5.0 \times 10^{-18}$ \\
\hline
\end{tabular}

Table 2 Point source fluxes to reach $\mathrm{S} / \mathrm{N}=3$ in $1000 \mathrm{sec}$ (spectroscopy)

\begin{tabular}{ll}
\hline Wavelength $(\AA)$ & Flux $\left(\mathrm{erg} \mathrm{s}^{-1} \mathrm{~cm}^{-2} \AA^{-1}\right)$ \\
\hline 1215 & $8.0 \times 10^{-15}$ \\
1306 & $6.0 \times 10^{-16}$ \\
1335 & $8.0 \times 10^{-16}$ \\
1550 & $1.0 \times 10^{-15}$ \\
2500 & $1.3 \times 10^{-16}$ \\
\hline
\end{tabular}

- Time resolution as short as 40 milliseconds to track the evolution of instabilities in discs around compact sources.

The sensitivity requirements are given in Tables 1 and 2 .

\section{ISSIS optical design and expected performances}

ISSIS optical bench assembly (without cover) is depicted in Fig. 1 (see also, Gómez de Castro et al. 2010, 2012a, 2012b, 2014).

The WSO-UV Fine Guiding Sensors (FGS) focus the telescope beam on the entrance slits of the spectrographs. In WSO-UV, ISSIS is located below the primary mirror of the telescope and above the optical bench. ISSIS captures the central part of the telescope beam with a mobile pick-off mirror that acts as a refocusing mechanism (RM). The RM is needed to fold and adapt the telescope beam to ISSIS optics. The focal surface of the T170M telescope in WSO-UV is below ISSIS, at the plane where the spectrographs entrance slits are. The FGS are also in the same plane. The RM acts on the intermediate image from the telescope before being sent to the first mirror of ISSIS optical relay. After the RM, there is a positive followed by a negative mirror $(\mathrm{M} 1+\mathrm{M} 2)$ to increase the focal length of the system; M1 is an even aspheric mirror with a positive radius of curvature and $\mathrm{M} 2$ is a toroidal mirror with a negative radius of curvature. The focal length of the T170M is $17 \mathrm{~m}$ and the focal length of ISSIS is $116.573 \mathrm{~m}$ providing a final focal ratio of 69 for ISSIS. Each ISSIS channel is able to operate in imaging mode, slitless spectroscopy mode or calibration mode through the use of a mode selection mechanism (MSM). On imaging mode, MSM uses flat mirrors aligned at such an angle that redirects the light towards one of the channels. Both channels contain a set of spectral filters that are accommodated in two sets of filter wheels (FWs) along the optical path. There will be

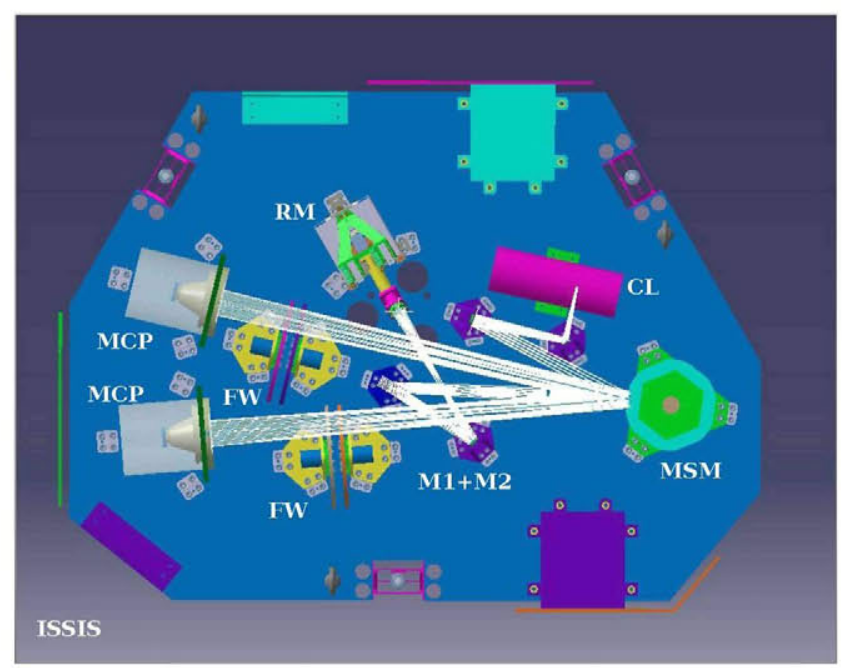

Fig. 1 Schematic view of ISSIS instrument. The letters indicate the main opto-mechanical elements described in the text. The acronyms stand for: refocusing mechanism $(R M)$, optical relay mirrors $(M 1$ and $M 2$ ), mode selection mechanism $(M S M)$, filter wheels $(F W)$, calibration lamp $(C L)$ and $\mathrm{MCP}$ detectors $(M C P)$

long-pass and narrow band filters for specific scientific investigations and, additionally, neutral density filters to allow observations of sources well above the sensitivity limit. ISSIS will be the only UV imager available in space for astronomical uses at the time of WSO-UV launch.

In slitless spectroscopy mode, the system makes use of flat reflective gratings as dispersive components; they are located on the MSM. There is one grating per channel to redirect the light either to the FUV or the NUV detectors. In nominal spectroscopic mode, the filter wheels are set in open (no-filter) position. The diffraction gratings have peak efficiency at $\sim 1400 \AA$ with groove density of 450 lines per $\mathrm{mm}$ for the FUV channel and at $\sim 2300 \AA$ with $250 \mathrm{l} / \mathrm{mm}$ for the NUV channel.

Finally, the calibration mode is used to perform flat-field calibration of the FUV and NUV detectors on a pixel-topixel basis (high-spatial frequency flat). In this mode, a shutter is used to block the light coming from the telescope. For calibration, the MSM is set in a configuration that contains a flat mirror aligned at such an angle that directs the light coming from a calibration subsystem into the end of the optical path of the FUV or NUV channel (from the FWs to the detectors). The calibration subsystem consists of a deuterium discharge lamp and two mirrors to project a uniform beam on the focal plane assembly. The main optical parameters for ISSIS are summarized in Table 3.

The total efficiency of ISSIS is characterized by the integrated system throughput. This throughput has been determined by taking into account the telescope performance, the internal reflections in the instrument and the quantum efficiencies of the MCP detectors, whereas filters transmittance has not been included in the throughput calculations. 
Table 3 Main characteristics of the ISSIS instrument

\begin{tabular}{lll}
\hline & FUV channel & NUV channel \\
\hline Spectral range & $1150-1750 \AA$ & $1850-3200 \AA$ \\
Peak throughput (imaging) & $\sim 1400 \AA$ & $\sim 2500 \AA$ \\
Field of view: imaging & $70 \operatorname{arcsec} \times 75 \operatorname{arcsec}$ & $70 \operatorname{arcsec} \times 75 \operatorname{arcsec}$ \\
Field of view: spectroscopy & $36 \operatorname{arcsec} \times 65 \operatorname{arcsec}$ & 31 arcsec $\times 61$ arcsec \\
Detector type & CsI MCP & CsTe MCP \\
Detector diameter & $40 \mathrm{~mm}$ & $40 \mathrm{~mm}$ \\
Detector format (equivalent) & $>2048 \times 2048$ pix & $>2048 \times 2048$ pix \\
Pixel scale & 0.036 arcsec & 0.036 arcsec \\
Scale ratio between & $<7 \%$ & $<7 \%$ \\
orthogonal axis & & 4 \\
Number of reflections & 4 & $40 \mathrm{~ms}$ \\
Temporal resolution & $40 \mathrm{~ms}$ & $R=500$ \\
Slitless spectroscopy resolution & $R=500$ &
\end{tabular}

The expected performance of ISSIS in imaging mode involves a total throughput of $\sim 1.2 \%$ at $\sim 1400 \AA$ (FUV channel) and $\sim 6.5 \%$ at $\sim 2500 \AA$ (NUV channel). For slitless spectroscopy we obtain throughput peaks of $\sim 0.3 \%$ $(1400 \AA)$ and $\sim 2.9 \%(2500 \AA)$ for FUV and NUV, respectively. The estimated sensitivity in this section is conservative and based on UVIT-alike detectors ${ }^{1}$ (Hutchings 2013). The final provider for ISSIS detectors is yet to be defined.

A comparison of the main characteristics of WSO-ISSIS, GALEX (Morrissey et al. 2007) and HST in the imaging and spectroscopy modes is performed. Table 4 shows the comparison of ISSIS, GALEX and HST (ACS/SBC, WFC3) in imaging mode (Space Telescope Science Institute 2014a) and Tables 5 and 6 show the comparison of ISSIS, GALEX and HST (STIS, COS) (Space Telescope Science Institute 2014c; Space Telescope Science Institute 2014b) in spectroscopy mode. Notice that the quoted spatial resolution corresponds to the PSF integrated over a 10-20 minutes exposure time. This includes a significant broadening caused by the accuracy of the FGS system; FGS guarantee a pointing accuracy better than 0.1 arcsec at 3 sigma (Shustov et al. 2014). This motion is susceptible to be used to decrease the MCP detector fatigue; see, for instance Martin et al. (2003) for the GALEX strategy for this purpose. Moreover, part of the optical quality loosed by the pointing inaccuracy can be recovered making use of the photon counting nature of MCPs detectors. As a result, ISSIS pixel scale has been selected to be $0.036 \mathrm{arcsec} / \mathrm{pix}$ though, naively, it may look as the ISSIS PSF being oversampled.

ISSIS performance is, in all senses, intermediate between the GALEX wide field imagers and the high sensitivity

\footnotetext{
${ }^{1}$ UVIT is a wide field imaging instrument for UV-Visible purposes to be launched by the Indian Space Agency in ASTROSAT.
}

HST instruments such as the Cosmic Origins Spectrograph (COS). In imaging mode, ISSIS is designed to be like the Solar Blind Channel (SBC) and the High Resolution Channel (HRC) in the Advanced Camera System (ACS) on board Hubble. However, ISSIS will improve on ACS spectroscopic capabilities making use of gratings instead of prisms. ISSIS is designed as an analysis tool of the UV sky unveiled by GALEX.

ISSIS features compared with GALEX can be summarized into: higher spatial resolution (about 30 times higher than GALEX resolution), simple spectroscopic mode with uniform dispersion and sensitivity below $\sim 1300 \AA$ (ISSIS short wavelength cut-off is set by the $\mathrm{MgF}_{2}$ short wavelength transmission cut-off, $\sim 1150 \AA$ ). Even thought ISSIS throughput in the FUV channel is below that of GALEX, the final ISSIS effective area is higher because of the larger collecting surface of the WSO-UV telescope. Nevertheless, WSO-ISSIS has an effective area smaller than the area for the HST instruments. In order to compare the photometric efficiencies we plot the effective areas for these instruments in Figs. 2 and 3. ISSIS detectors contract has not been yet issued and their performance for the plots has been extrapolated using as baseline the detectors for the UVIT mission. As shown in the figures, ISSIS sensitivity compares well with HST instruments in the NUV range with this type of detectors; however, their FUV performance can be improved.

\section{Provisions for the performance verification of ISSIS}

As usual, ISSIS tests will proceed at the three levels: components, ISSIS at subsystems and system level and final tests with ISSIS integrated in WSO-UV (see scheme in Fig. 4). At the component level, the tests are defined as follow: 
Table 4 Comparison of the main characteristics and performances of the ISSIS, GALEX and HST instruments in imaging mode

\begin{tabular}{|c|c|c|c|c|c|c|}
\hline \multirow[t]{2}{*}{ CHANNEL } & \multicolumn{2}{|l|}{ ISSIS } & \multicolumn{2}{|l|}{ GALEX } & \multicolumn{2}{|l|}{ HUBBLE } \\
\hline & FUV & NUV & FUV & NUV & $\mathrm{SBC}(\mathrm{ACS})$ & WFC3 (UVIS) \\
\hline SPECTRAL RANGE & $1150-1750 \AA$ & $1850-3200 \AA$ & $1344-1786 \AA$ & $1771-2831 \AA$ & $1150-1700 \AA$ & $2000-10000 \AA$ \\
\hline PEAK THROUGHPUT & $\sim 1400 \AA$ & $\sim 2500 \AA$ & $\sim 1516 \AA$ & $\sim 2200 \AA$ & $\sim 1250 \AA$ & $\sim 6000 \AA$ \\
\hline PEAK EFFICIENCYa & $1.2 \%$ & $6.5 \%$ & $1.8 \%$ & $3.1 \%$ & $7.5 \%$ & $27 \%$ (including filter transmission) \\
\hline FIELD OF VIEW & $70 \operatorname{arcsec} \times 75 \operatorname{arcsec}$ & $70 \operatorname{arcsec} \times 75 \operatorname{arcsec}$ & $4572 \operatorname{arcsec}$ & $4500 \operatorname{arcsec}$ & $34.6 \operatorname{arcsec} \times 30.8 \operatorname{arcsec}$ & $162 \operatorname{arcsec} \times 162 \operatorname{arcsec}$ \\
\hline DETECTOR FORMAT & $\begin{array}{l}2048 \times 2048 \text { pix } \\
\text { CsI MCP }\end{array}$ & $\begin{array}{l}2048 \times 2048 \text { pix } \\
\text { CsTe MCP }\end{array}$ & $\begin{array}{l}3840 \times 3840 \text { pix } \\
\text { CsI MCP }\end{array}$ & $\begin{array}{l}3840 \times 3840 \text { pix } \\
\text { Cs2Te MCP }\end{array}$ & $\begin{array}{l}1024 \times 1024 \text { pix } \\
\text { CsI MCP }\end{array}$ & $\begin{array}{l}2 \times 2051 \times 4096 \text { pix } \\
\text { CsTe MCP }\end{array}$ \\
\hline PIXEL SCALE & $0.036 \mathrm{arcsec} / \mathrm{pix}$ & $0.036 \mathrm{arcsec} / \mathrm{pix}$ & $1.5 \mathrm{arcsec} / \mathrm{pix}$ & $1.5 \mathrm{arcsec} / \mathrm{pix}$ & $0.034 \times 0.030 \mathrm{arcsec} / \mathrm{pix}$ & $0.039 \times 0.039 \mathrm{arcsec} / \mathrm{pix}$ \\
\hline $\begin{array}{l}\text { SPATIAL RESOLUTION } \\
\text { (FWHM) }\end{array}$ & $0.15 \operatorname{arcsec}$ & $0.15 \operatorname{arcsec}$ & $4.3 \operatorname{arcsec}$ & $5.3 \operatorname{arcsec}$ & $0.077 \times 0.066 \operatorname{arcsec}$ & $0.07 \operatorname{arcsec}$ \\
\hline
\end{tabular}

${ }^{\mathrm{a}}$ Based on UVIT detectors sensitivity 
Table 5 Comparison of the main characteristics and performances of the ISSIS, GALEX and HST instruments in spectroscopy mode in the FUV channel

\begin{tabular}{|c|c|c|c|c|}
\hline & ISSIS & GALEX & STIS HUBBLE & COS HUBBLE \\
\hline CHANNEL & FUV & FUV & FUV-MAMA & FUV-MAMA \\
\hline SPECTRAL RANGE & $1150-1750 \AA$ & $1344-1786 \AA$ & $1150-1700 \AA$ & $900-2150 \AA$ \\
\hline PEAK THROUGHPUT & $\sim 1400 \AA$ & $\sim 1516 \AA$ & $\sim 1216 \AA$ & $\sim 1335 \AA$ \\
\hline PEAK EFFICIENCYa & $0.3 \%$ & $1.0 \%$ & $3.4 \%(\mathrm{G} 140 \mathrm{~L})$ & $3.4 \%(\mathrm{G} 140 \mathrm{~L})$ \\
\hline FIELD OF VIEW & $36 \operatorname{arcsec} \times 65 \operatorname{arcsec}$ & $4572 \operatorname{arcsec}$ & $25 \operatorname{arcsec} \times 25 \operatorname{arcsec}$ & $25 \operatorname{arcsec} \times 25 \operatorname{arcsec}$ \\
\hline DETECTOR FORMAT & $\begin{array}{l}2048 \times 2048 \text { pix } \\
\text { CsI MCP }\end{array}$ & $\begin{array}{l}3840 \times 3840 \text { pix } \\
\text { CsI MCP }\end{array}$ & $\begin{array}{l}1024 \times 1024 \text { pix } \\
\text { CsI MCP }\end{array}$ & $\begin{array}{l}2 \times 16384 \times 1024 \text { pix } \\
\text { CsI MCP }\end{array}$ \\
\hline PIXEL SCALE & $0.036 \mathrm{arcsec} / \mathrm{pix}$ & $1.5 \mathrm{arcsec} / \mathrm{pix}$ & $0.0245 \times 0.0247 \mathrm{arcsec} / \mathrm{pix}$ & $0.114 \mathrm{arcsec} / \mathrm{pix}$ \\
\hline SPECTRAL RESOLUTION & 500 & $250-300$ & $500-10010$ at $1150-3100 \AA$ & $16000-24000$ med $2000-3000$ low \\
\hline DETECTORS EFFICIENCY & $8 \%$ at $1400 \AA$ & $12 \%$ at $1516 \AA$ & $24 \%$ at $1216 \AA$ & $26 \%$ at $1335 \AA$ \\
\hline
\end{tabular}

${ }^{a}$ Based on UVIT detectors sensitivity

Table 6 Comparison of the main characteristics and performances of the ISSIS, GALEX and HST instruments in spectroscopy mode in the NUV channel

\begin{tabular}{lllll}
\hline & ISSIS & GALEX & STIS HUBBLE & COS HUBBLE \\
\hline CHANNEL & NUV & NUV & NUV-MAMA & NUV-MAMA \\
SPECTRAL RANGE & $1850-3200 \AA$ & $1771-2831 \AA$ & $1600-3100 \AA$ & $1650-3200 \AA$ \\
PEAK THROUGHPUT & $\sim 2500 \AA$ & $\sim 2200 \AA$ & $\sim 2537 \AA$ & $\sim 2250 \AA$ \\
PEAK EFFICIENCY & $2.9 \%$ & $2.1 \%$ & $1.7 \%(\mathrm{G} 230 \mathrm{~L})$ & $1.5 \%(\mathrm{G} 230 \mathrm{~L})$ \\
FIELD OF VIEW & $31 \operatorname{arcsec} \times 61$ arcsec & $4500 \operatorname{arcsec}$ & $25 \operatorname{arcsec} \times 25$ arcsec & 25 arcsec $\times 25$ arcsec \\
DETECTOR FORMAT & $2048 \times 2048$ pix & $3840 \times 3840$ pix & $1024 \times 1024$ pix & $1024 \times 1024$ pix \\
& CsTe MCP & Cs2Te MCP & CsTe MCP & CsTe MCP \\
PIXEL SCALE & 0.036 arcsec/pix & $1.5 \operatorname{arcsec} / \mathrm{pix}$ & $0.0245 \times 0.0248$ arcsec/pix & 0.0235 arcsec/pix \\
SPECTRAL RESOLUTION & 500 & $80-150$ & $500-10010$ at $1150-3100 \AA$ & $16000-24000 \mathrm{med} 2000-3000$ low \\
DETECTORS EFFICIENCY & $17 \%$ at $2300 \AA$ & $8 \%$ at $2200 \AA$ & $9 \%$ at $2537 \AA$ & $10 \%$ at $2250 \AA$ \\
\hline
\end{tabular}

${ }^{a}$ Based on UVIT detectors sensitivity

- Detectors: The tests performed on the detector could include: sensitivity, response as a function of wavelength, spatial variation of sensitivity, tests to estimate the centroid of the detected photons, photometric non-linearity in photon-counting mode, gain as a function of MCP voltage, distortions on the detector. Dark rate, dynamic range, and cosmetic defect fraction will be measured. While some of these measurements will be repeated often during the course of subsystem testing and instrument integration, definitive measurements must be made in the final configuration under realistic environmental conditions, in the instrument level tests phase.

- Filters: The transmission of each filter as a function of wavelength, spatial variation of sensitivity with position as a function of wavelength, shift in focus due to the filters.
- Mirrors: Reflectivity as a function of wavelength, spatial variation of sensitivity with position as a function of wavelength, measure of the focus position of the mirrors.

- Gratings: Efficiency, wavelength range, length of the spectra on the detector plane, shift between the object position in the image and the 0th-order spectrum, scattered and stray-light tests.

- Calibration Lamps: The main goal is to measure and characterize the global count-rates of the internal deuterium (D2) calibration lamp through the filters. Degradation in the lamp's output with time will be also investigated for a fixed time period.

For ISSIS tests at instrument level, it is required to develop new facilities for vacuum testing. As today, there are several possibilities being analyzed in Spain and possibly in Russia. Basic verification tests are: geometric tests, geometric distortion tests, image quality tests, radiometric efficiency, spectroscopy tests, uniformity of the radiometric re- 
Fig. 2 Comparison between the effective area in logarithmic scale for ISSIS, GALEX and $\mathrm{SBC}$ in imaging mode
Fig. 3 Comparison between the effective areas for ISSIS, GALEX and HST (STIS and COS) in spectroscopy mode
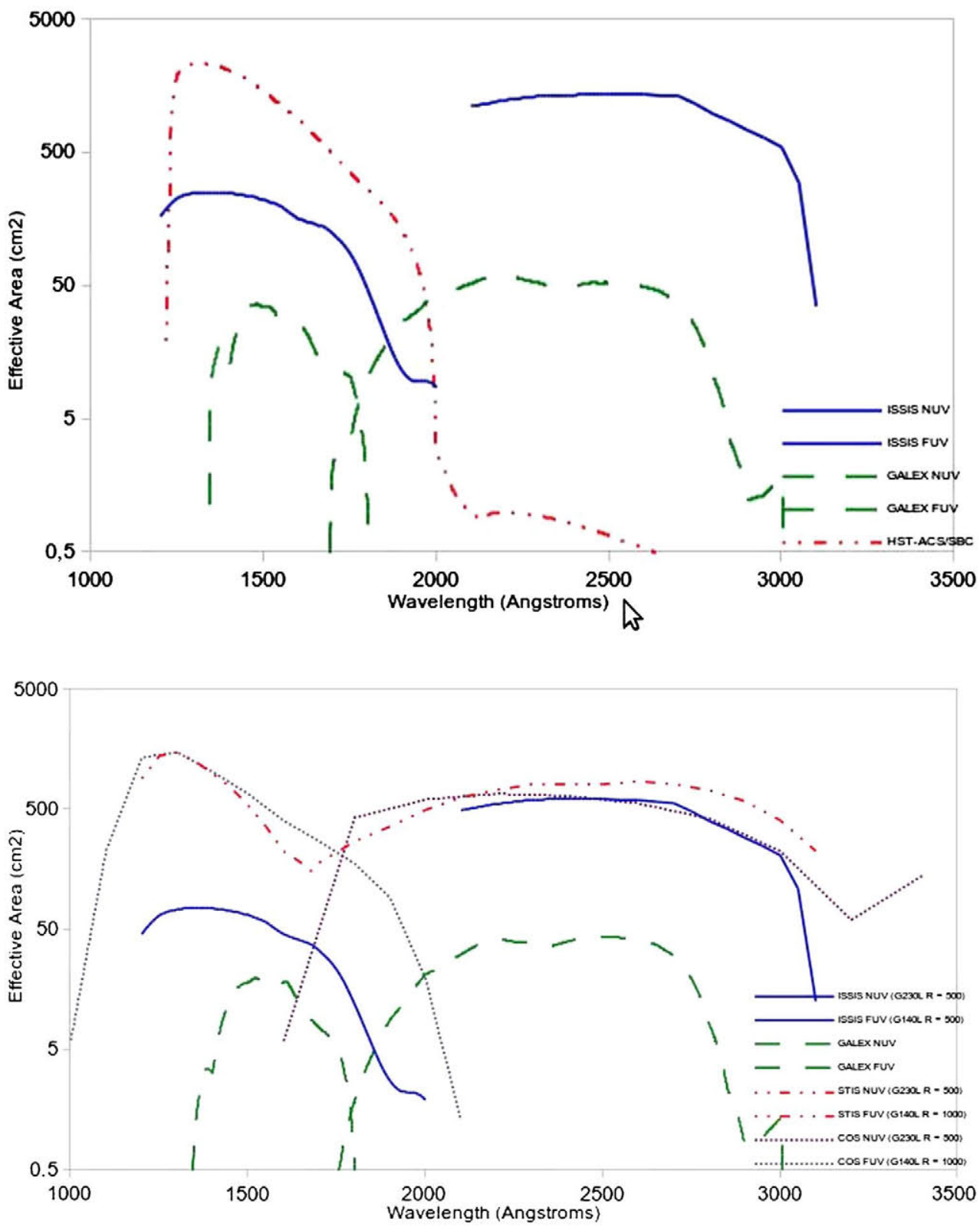

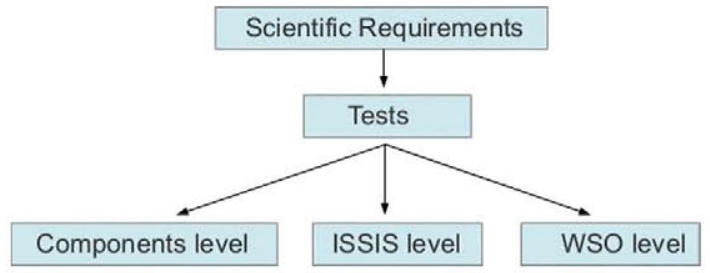

Fig. 4 Schematic diagram for ISSIS verification tests

sponse, exposure time repeatability, stray-light tests, mechanism functionality and repeatability. The main challenges for performing the tests of ISSIS involve the development of a vacuum chamber fully equipped for UV optical tests and the telescope simulator for the on-axis beam.
A major source of concern during ISSIS integration, launch and in-flight performance is contamination by molecules and particles of the relevant surfaces (detectors windows and optical elements). Contamination affects not only to the quality of the image (wavefront) but also to the radiometry of the instrument. Moreover, molecular contaminants polymerize with UV light into knotty blobs, rather than in uniform layers introducing all sort of features in the images. An estimate of ISSIS radiometric capabilities degradation by molecular contamination is shown in Fig. 5 being ISSIS molecular contamination level per optical surface lower than $\sim 15 \AA$. Threshold requirement is equivalent to $\sim 15 \%$ radiometric degradation al Ly-alpha per surface. This requirement is roughly equivalent to $1.5 \times 10^{-7} \mathrm{~g} / \mathrm{cm}^{2}$ during the mission lifetime. 
Fig. 5 Estimated ISSIS radiometric efficiency loss by molecular contamination as a function of the thickness of the molecular film

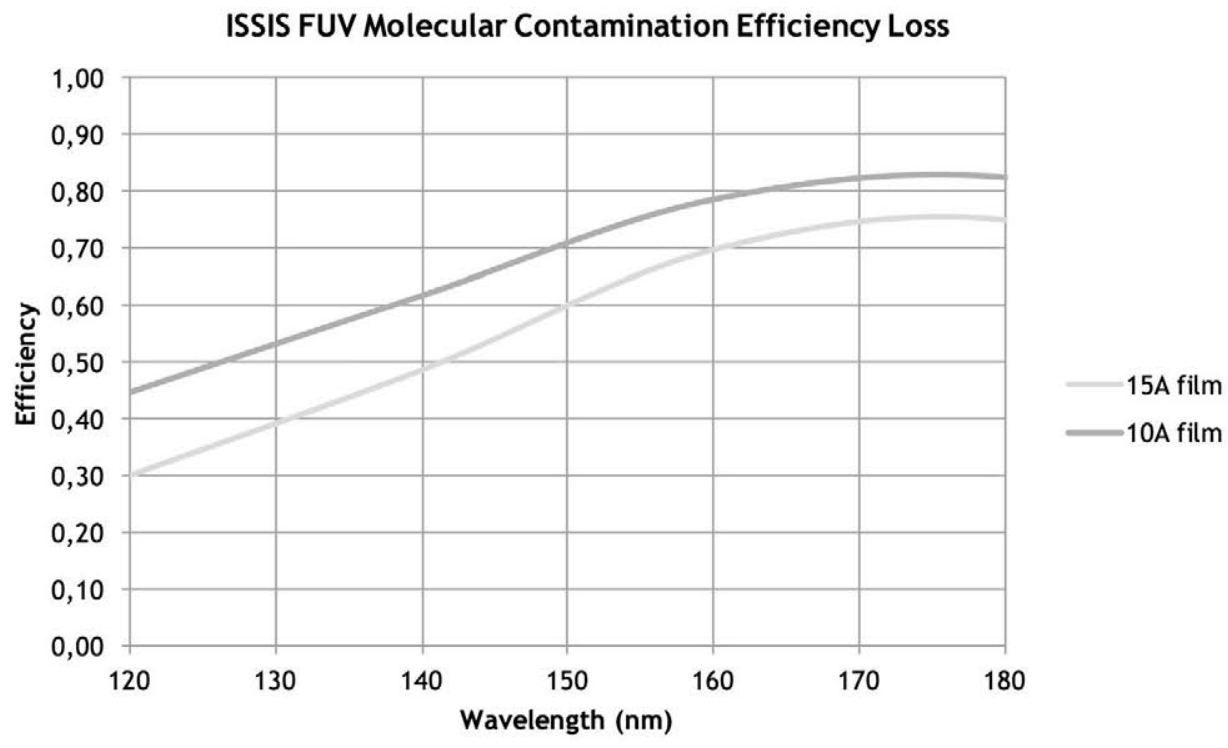

ISSIS NUV Molecular Contamination Efficiency Loss

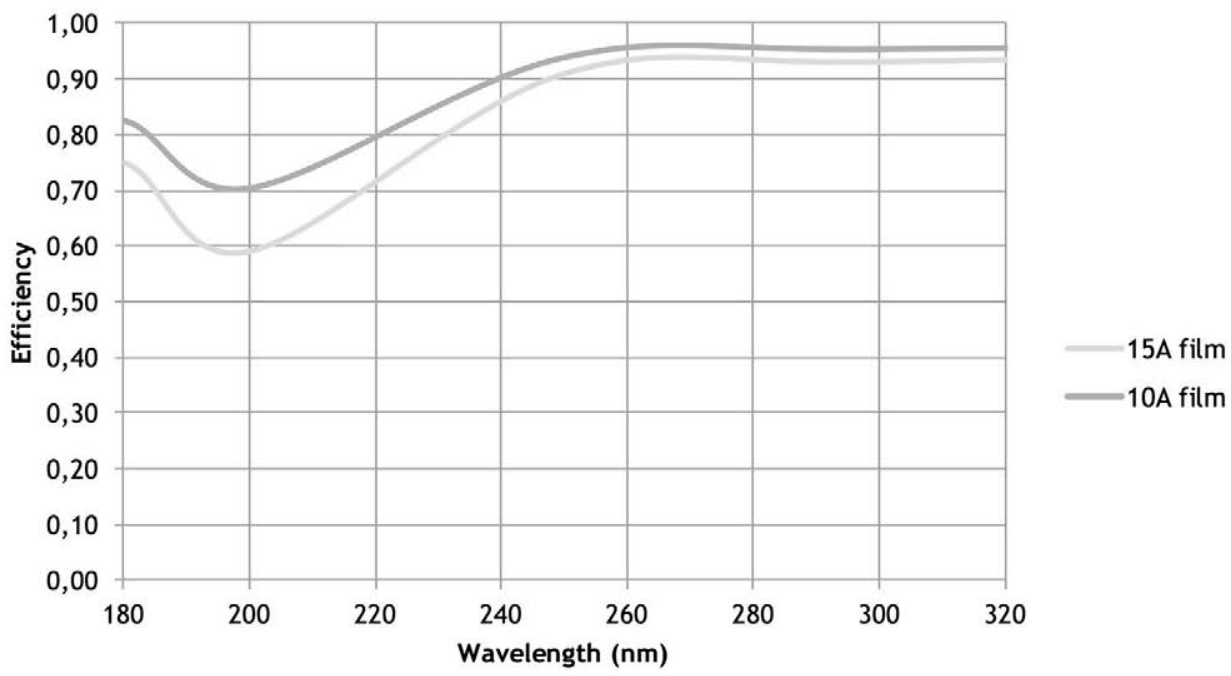

Following the precedent of the SOHO mission (Schuehle 2003), a cleanliness program will be set to ensure stable radiometric performance. Thus, cleanliness requirements are to be defined for instruments and spacecraft (the latter mostly based on the requirements set by the instruments). A Cleanliness Control Plan will govern all activities related to cleanliness and contamination. The SOHO team gathered information that will be used to define ISSIS and WSO-UV cleanliness control plan, very especially the procedures they developed to derive and implement the cleanliness requirements, and how they are monitored and verified.

\section{ISSIS basic operation modes}

The main operation modes of ISSIS are enumerated below (Perea and Gómez de Castro 2013):
1. FUV Imaging Accumulation Mode (FUV/IMA).

2. FUV Imaging Time-Tag Mode (FUV/IMA-Time-TAG).

3. FUV Spectroscopy Accumulation Mode (FUV/SPEC).

4. FUV Spectroscopy Time-Tag Mode (FUV/SPEC-TimeTAG).

5. NUV Imaging Accumulation Mode (NUV/IMA).

6. NUV Imaging Time-Tag Mode (NUV/IMA-Time-TAG).

7. NUV Spectroscopy Accumulation Mode (NUV/SPEC).

8. NUV Spectroscopy Time-Tag Mode (NUV/SPEC-TimeTAG).

Photon events will be processed on board and centroids will be determined and stored. In TIME-TAG mode, the precise event timing will be stored with a relative accuracy of $40 \mathrm{~ms}$ but only over reduced fields in the detector being photon counting image mode on $10 \times 10$ pixels on the detector and photon counting slitless spectroscopy mode on $10 \times 300$ pixels on the detector. In ACCUM mode, 
the full detector will be available but the events will be coadded for a single pixel and stored (Gómez de Castro 2012; Kumar et al. 2012).

ISSIS will also be operated in CORONOGRAPHIC mode. This mode is defined to alleviate the detector fatigue when observing faint structures close to bright sources. These dead areas on the detector could be implemented with masks in the photo-cathode. There are still on-going studies on the best strategies to set these few dead-areas.

Finally, there will also be a CALIBRATION mode to measure the degradation of the pixel-to-pixel (small scale) response making use of the deuterium lamp. Photometric and spectroscopic calibrations will be carried out based on astronomical sources making use of the information in the NASA Multimission Archive on the HST and GALEX mission. ISSIS spectroscopic mode is very similar to the low dispersion mode in the International Ultraviolet Explorer (IUE). Observations from the IUE INES Final Archive will be used for this purpose.

\section{Data handling strategies}

ISSIS data policy will be to create final products in format similar to high-energy photon counting missions. In the photon-counting mode, ISSIS will not provide an integrated, full-frame two-dimensional image but a list of events corresponding to the time of arrival of each photon. The resulting file will be a FITS binary table with information about the arrival time of the photon, its position in the detector and a number of flags that will account for the quality of the measurement and other issues, such as the possible fraction of pile-up. This policy is similar to that of current missions like XMM-Newton and will offer an opportunity to the high-energy community to use the same analytic tools they are currently managing for their data. It is important to notice that the photon-counting mode does not mean a loss of imaging information since an image can be constructed in any case by accumulating the photons during the whole exposure time. In ACCUM mode, data will be provided as standard FITS images.

\section{Conclusions}

ISSIS compares well with the previous UV instrumentation flown in the NASA missions GALEX and HST. The spectroscopic mode has been defined to provide uniform dispersion and photon counting capabilities to study weak sources such as young planetary systems, solar-like stars and distant active sources. The coronographic capabilities will make of ISSIS a unique instrument to resolve jets from their parent sources and study the impact of UV radiation in the young planetary disks evolution.
From the technical point of view, ISSIS main challenges are the procurement of high sensitivity FUV detectors and the development of the facilities/team for the Assembly, Test and Verification in close coordination with the Russian WSO-UV team.

WSO-UV will be launched in this decade and it will be a fundamental tool for the development of astronomical knowledge. WSO-UV will open new opportunities in planetary science, stellar astrophysics, extragalactic astronomy and cosmology. With its launch date, WSO is ideally placed to provide follow-up studies of the large number of UV sources found by the GALEX sky survey after the end of the HST mission.

Acknowledgements ISSIS development is being funded by Ministry of Industry, Tourism and Commerce of Spain. We acknowledge the Russian WSO-UV team for their support in this paper. The science team at Universidad Complutense de Madrid acknowledges the financial support of the Ministry of Economy and Competitiveness through grants AYA2008-06423-C03-01 and AYA2011-29754-C03-C01.

\section{References}

Gómez de Castro, A.I.: System Requirements Specification (2012) Gómez de Castro, A.I., Shustov, B., Sachkov, M., Kappelmann, N., Huang, M., Werner, K.: The space telescope for ultraviolet astronomy wso-uv, p. 219 (2010)

Gómez de Castro, A.I., Sanchez, N., Sestito, P., Rodriguez, P., Gomez, M.T., Seijas, J., Lopez-Martinez, F., Quintana, J., Ubierna, M., Muñoz, J.: Society of Photo-Optical Instrumentation Engineers (SPIE) Conference Series, vol. 8443. SPIE Press, Bellingham (2012a)

Gómez de Castro, A.I., Sánchez Doreste, N., Paola, S., et al.: Editorial Complutense, Madrid (2012b)

Gómez de Castro, A.I., Sestito, P., Sánchez, N., López-Martínez, F., Seijas, J., Gómez, M., Rodríguez, P., Quintana, J., Ubierna, M., Muñoz, J.: Adv. Space Res. 53, 996 (2014)

Hermanutz, S., Barnstedt, J., Diebold, S., Elsener, H.R., Ganz, P.R., Kalkuhl, C., Kappelmann, N., Pfeifer, M., Tanirah, O., Sachkov, M., Schaadt, D.M., Schanz, T., Shustov, B.M., Werner, K.: In: Society of Photo-Optical Instrumentation Engineers (SPIE) Conference Series, vol. 8443. SPIE Press, Bellingham (2012)

Hutchings, J.B.: Challenges in UV Astronomy, ESO Garching (2013)

Kumar, A., Ghosh, S.K., Hutchings, J., Kamath, P.U., Kathiravan, S., Mahesh, P.K., Murthy, J., Nagbhushana, S., Pati, A.K., Rao, M.N., Rao, N.K., Sriram, S., Tandon, S.N.: In: Society of PhotoOptical Instrumentation Engineers (SPIE) Conference Series, vol. 8443. SPIE Press, Bellingham (2012)

Martin, C., Barlow, T., Barnhart, W.B., et al.: Future EUV/UV and visible space astrophysics missions and instrumentation. In: Blades, J.C., Siegmund, O.H.W. (eds.) Proceedings of the SPIE, vol. 4854, pp. 336-350 (2003)

Morrissey, P., Conrow, T., Barlow, T.A., Small, T., Seibert, M., Wyder, T.K., Budavári, T., Arnouts, S., Friedman, P.G., Forster, K., Martin, D.C., Neff, S.G., Schiminovich, D., Bianchi, L., Donas, J., Heckman, T.M., Lee, Y.-W., Madore, B.F., Milliard, B., Rich, R.M., Szalay, A.S., Welsh, B.Y., Yi, S.K.: Astrophys. J. Suppl. Ser. 173, 682 (2007)

Perea, G.B., Gómez de Castro, A.I.: Operational modes of ISSIS for the Electrical Model (2013)

Sachkov, M.: Astrophys. Space Sci. 329, 261 (2010) 
Sachkov, M., Shustov, B., Gómez de Castro, A.I.: Adv. Space Res. 53, $990(2014)$

Schuehle, U.H.: Cleanliness and calibration stability of uv instruments on soho, vol. 4853, p. 88 (2003)

Shustov, B., Gómez de Castro, A.I., Sachkov, M., et al.: (2014, to be published)
Space Telescope Science Institute: ACS: Surveys Instrument Handbook for Cycle, 22, vol. 13.0 (2014a)

Space Telescope Science Institute: COS: Surveys Instrument Handbook for Cycle, 22, vol. 6.0 (2014b)

Space Telescope Science Institute: STIS: Surveys Instrument Handbook for Cycle, 22, vol. 13.0 (2014c) 